\title{
Partial trisomy 7p defined by analysis of a complex chromosome rearrangement using a BAC clone panel
}

\author{
Cathy M. Tuck-Muller, $P h D^{1}$, Barbara K. Goodman, $P h D^{2}$, Shibo Li, $M D^{1}$, José E. Martinez, $M D^{1}$, \\ Xiao-Ning Chen, $M D^{3}$, Wladimir Wertelecki, $M D^{1}$, Julie R. Korenberg, $M D, P h D^{3}$, and Gail Stetten, $P h D^{2}$
}

Purpose: To illustrate the use of bacterial artificial chromosome (BAC) clone panels for molecular cytogenetic
analysis of complex chromosome rearrangements (CCRs). Methods: High resolution cytogenetics followed by
fluorescence in situ hybridization (FISH) analysis using chromosome band-specific BAC probes, in addition to
commercially available probes. Results: High resolution cytogenetics in conjunction with FISH using commercially
available probes proved inadequate to resolve problems in characterizing a balanced CCR in the mother of a
patient who had inherited an unbalanced form of the CCR. Accurate interpretation of the CCR and the
unbalanced rearrangement in the patient as trisomy $7 \mathrm{p} 12.2 \rightarrow \mathrm{p} 21.3$ was accomplished only through use of the
BAC clone panel. Conclusion: Use of BAC clone panels can enhance the power of FISH analysis in defining
chromosome rearrangements that cannot be resolved by high resolution chromosome analysis. Genetics in

Medicine, 2001:3(2):126-131.

Key Words: insertion(2;7), duplication 7p, inversion(7), FISH, BAC clones

Balanced complex chromosomal rearrangements (CCRs) usually come to medical attention either through unbalanced offspring or infertility. Because of their complexity, such rearrangements are often difficult to characterize unequivocally based on routine banding analysis alone. Accurate characterization of the CCR can be especially important for the prognosis and management of living unbalanced offspring.

While fluorescence in situ hybridization (FISH) analysis has proved a valuable adjunct to routine banding for accurate characterization of these rearrangements, ${ }^{1}$ the correct probes for analysis of many CCRs are not commercially available. This is illustrated by the CCR involving chromosomes 2 and 7 reported here, which was found in the phenotypically normal mother of a mentally retarded, dysmorphic girl. The patient had inherited the derivative chromosome (der) 2 but not the der 7 from her mother, resulting in an unbalanced karyotype.

High-resolution G-banding in conjunction with FISH analysis using commercially available probes proved insufficient to accurately characterize the CCR. Full characterization of this CCR was accomplished only through the use of a panel of locus-specific bacterial artificial chromosome (BAC) probes

\footnotetext{
From the ${ }^{I}$ Department of Medical Genetics, University of South Alabama, Mobile, Alabama; ${ }^{2}$ Department of Gynecology and Obstetrics, Johns Hopkins University, Baltimore, Maryland; ${ }^{3}$ Medical Genetics Birth Defects Center, Cedars-Sinai Medical Center and Departments of Pediatrics and Human Genetics, University of California at Los Angeles, Los Angeles, California.

Cathy M. Tuck-Muller, PhD, Department of Medical Genetics, University of South Alabama, CCCB 286, 307 University Boulevard, Mobile, AL 36688-0002.

Received: October 13, 2000.

Accepted: December 7, 2000
}

mapping to chromosome $7 \mathrm{p} 13 \rightarrow \mathrm{q} 22$. FISH analysis using this BAC clone panel permitted clear interpretation of the CCR in the patient's mother and, more importantly, conclusive identification of the chromosomal imbalance in the patient as partial trisomy 7p. Accurate characterization of the partial trisomy facilitated phenotypic comparisons to similar cases reported in the literature and led to a better patient management.

\section{CLINICAL REPORT}

The patient is a 13-year-old white female who was evaluated in our clinic because of mental retardation and dysmorphic features. She was born to healthy, unrelated parents and was the last of five pregnancies. Two of these pregnancies were miscarried and one infant had died at 2 days of age with coarctation of the aorta. Another pregnancy had produced a healthy male. The patient had a birth weight of $4,500 \mathrm{~g}$ after an unremarkable pregnancy. She was soon noted to be hypotonic and to have unusual physical features, but no further work up was pursued at that time. She has had no significant medical problems.

Physical examination at age 13 years revealed a short and dysmorphic girl with a head circumference of $53 \mathrm{~cm}$ (50th centile), height of $150 \mathrm{~cm}$ (10th centile), and weight of $61 \mathrm{~kg}$ (90th centile). Craniofacial dysmorphism included long facies with ocular hypertelorism, down-slanting palpebral fissures, prominent nasal bridge, large nose with columnella and nasal tip extending beyond hypoplastic alae nasi, large central incisors, low set and dysplastic ears, and high narrow palate (Fig. 1a and b). Micrognathia, which had been noted in an earlier evaluation at age 11, became less apparent over time and was not 
noted at age 13. She had hyperlaxity of elbows and interphalangeal joints, as well as small dysplastic nails. Dermatoglyphic analysis showed a normal finger tip pattern characterized by whorls (3), double loops (2), and ulnar loops (5) with hypoplastic dermal ridges and increased total ridge count of 246 (mean for normal females $=127$ ). A short third metatarsal bone bilaterally (Fig. 1c) and plantar vertical furrows (Fig. 1d) were also noted. No cardiovascular abnormalities were detected by physical examination. She had normal axillary and pubic hair, normal breast development (Tanner SMR III), and normal menstrual cycles. Neurologic examination was significant for universal hypotonia and poor motor coordination. No imaging studies were performed.

\section{MATERIALS AND METHODS}

\section{Cytogenetics}

High resolution chromosomes for GTG-banding analysis were prepared from synchronized peripheral blood lympho-

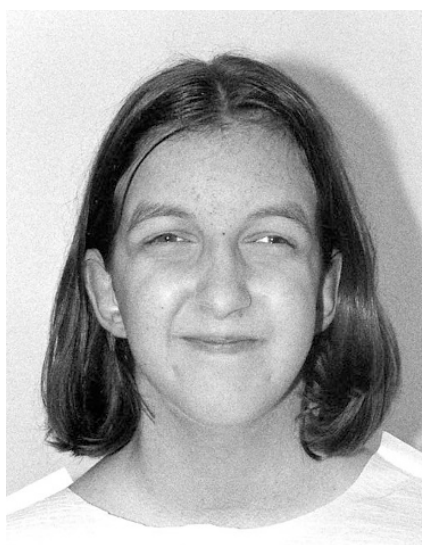

a
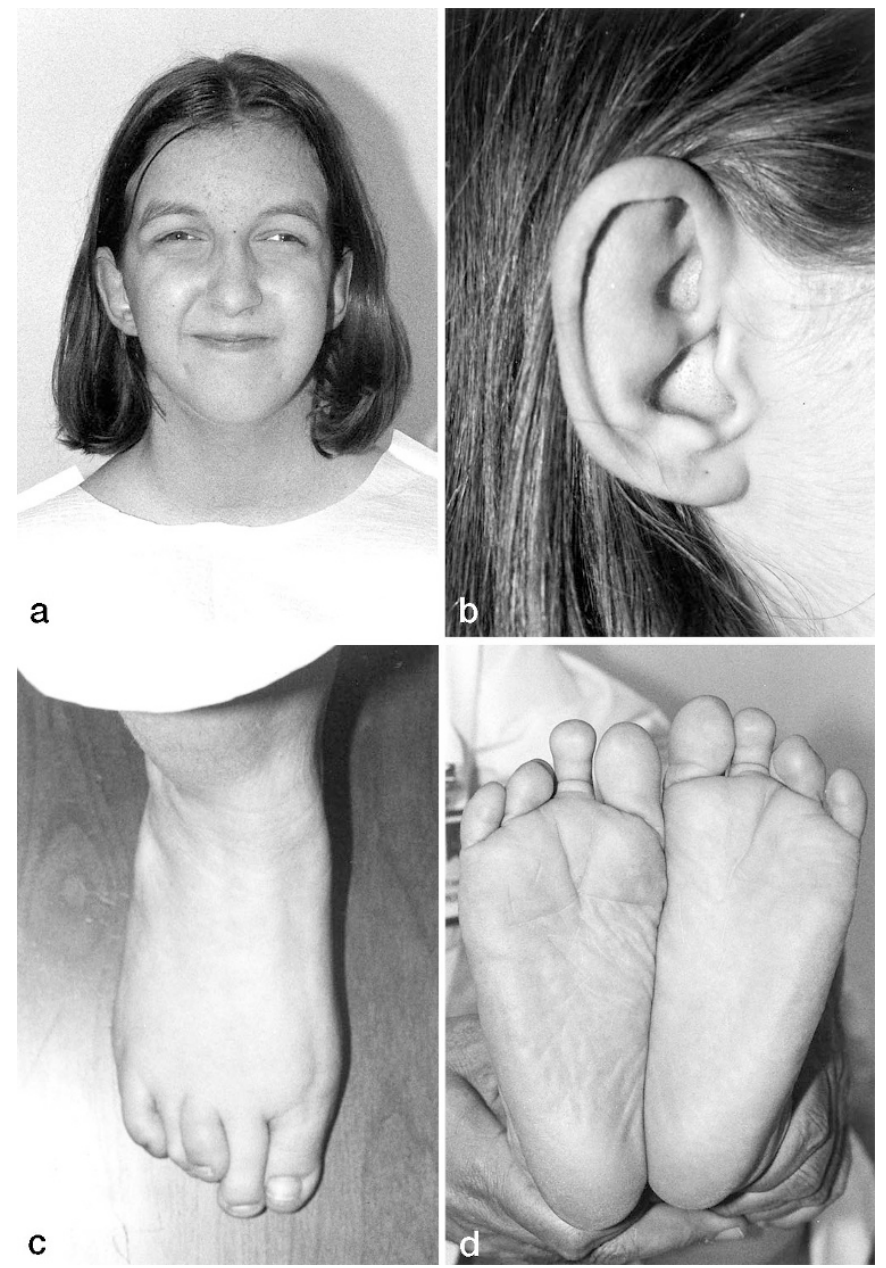

Fig. 1 Patient at age 13 years and 5 months showing a long face with long and down slanting palpebral fissures, strabismus, high nasal bridge and prominent jaw (a); patient ear showing prominent antihelix and dysplastic concha (b); right foot with overriding third toe secondary to a short third metatarsal bone (c); bilateral vertical plantar furrows; short, overriding third toes are hidden in this view (d). cytes $^{2}$ from the patient, her parents, and her maternal grandparents. For verification of the cytogenetic map location of BAC probes, synchronized peripheral blood lymphocytes from normal individuals were prepared by standard methods, applied to microscope slides, and stored at $-20^{\circ} \mathrm{C}$.

\section{Fluorescence in situ hybridization}

FISH studies were performed using painting probes specific for chromosomes 2 and 7 (WCP2 and WCP7, both from Vysis) and chromosome 8 (Coatosome 8 from Oncor). Commercial locus-specific probes used were D7S589 mapping to $7 \mathrm{p} 22$, WSCR mapping to $7 \mathrm{q} 11.23$ with control probe D7S427 mapping to 7q36, all from Oncor. The following BAC clones that map to chromosome 7 were also used: BAC RG180O01 mapping to $7 \mathrm{p} 21.1 \rightarrow \mathrm{p} 21.2$ and cross-hybridizing to the proximal long arm of chromosome 2 (Research Genetics, CITB library); and BACs 7B8 (403D01) mapping to $7 \mathrm{p} 15.3 \rightarrow \mathrm{p} 21.1,7 \mathrm{C} 13$ (403A08) mapping to $7 \mathrm{p} 13,7 \mathrm{~F} 3$ (009F05) mapping to 7q11.23 $\rightarrow \mathrm{q} 21.1$, 7F6 (402G05) mapping to 7q21.2, and 7F13 (666G06) mapping to 7q22 (all from the Cal Tech library CITB-978SK-A, provided by the BAC/PAC Molecular Cytogenetics Resource for Human Genetics, J.R. Korenberg and X-N. Chen, Cedars-Sinai Research Institute, Los Angeles). BAC 7F13 contains the STS D7S666. CEP 7 (Vysis), a direct-labeled commercial probe for the centromere of chromosome 7, was used for chromosome identification with some BACs.

Probes purchased from Oncor and Vysis were used according to the instructions of the manufacturer with minor modifications. Preparation of BAC DNA has been described previously. ${ }^{3}$ One microgram of BAC DNA was labeled with either biotin (BRL) or digoxigenin (Boehringer Mannheim) by nick translation (BRL). For each hybridization, an aliquot (200 ng) of labeled probe was coprecipitated with $5 \mu \mathrm{g}$ of human Cot- 1 DNA, then resuspended in $10 \mu \mathrm{L}$ of $50 \%$ formamide in $2 \mathrm{XSSC}$, $10 \%$ dextran sulfate. In some instances, as an internal control for identification of chromosome 7, $1 \mu \mathrm{L}$ of direct-labeled (Spectrum Green) CEP 7 alpha-satellite probe (Vysis) was added to the hybridization mixture. The probe solution was denatured for 5 minutes at $75^{\circ} \mathrm{C}$ and preannealed at $37^{\circ} \mathrm{C}$ for 40 minutes. Slides were denatured for 3 minutes at $75^{\circ} \mathrm{C}$ before hybridization at $37^{\circ} \mathrm{C}$ for 18 hours. The slides were washed in two changes each of $50 \%$ formamide/2XSSC ( 7 minutes) and 2XSSC ( 5 minutes) at $42^{\circ} \mathrm{C}$, and $4 \mathrm{XSSC} / 0.05 \%$ Tween-20 (1 minute) at room temperature. The BAC signals were detected using Texas Red-conjugated avidin (for biotinylated probes) and/or FITC-conjugated antidigoxigenin, as appropriate, according to manufacturer's instructions (Ventana, Inc.). Chromosomes were counterstained with the A-T binding fluorochrome 4',6'-diamidino-2'-phenylindole (DAPI) and viewed using a Zeiss AxioSkop equipped with a SenSys cooled CCD camera (Photometrics) and Quips SmartCapture VP imaging software (Vysis). 


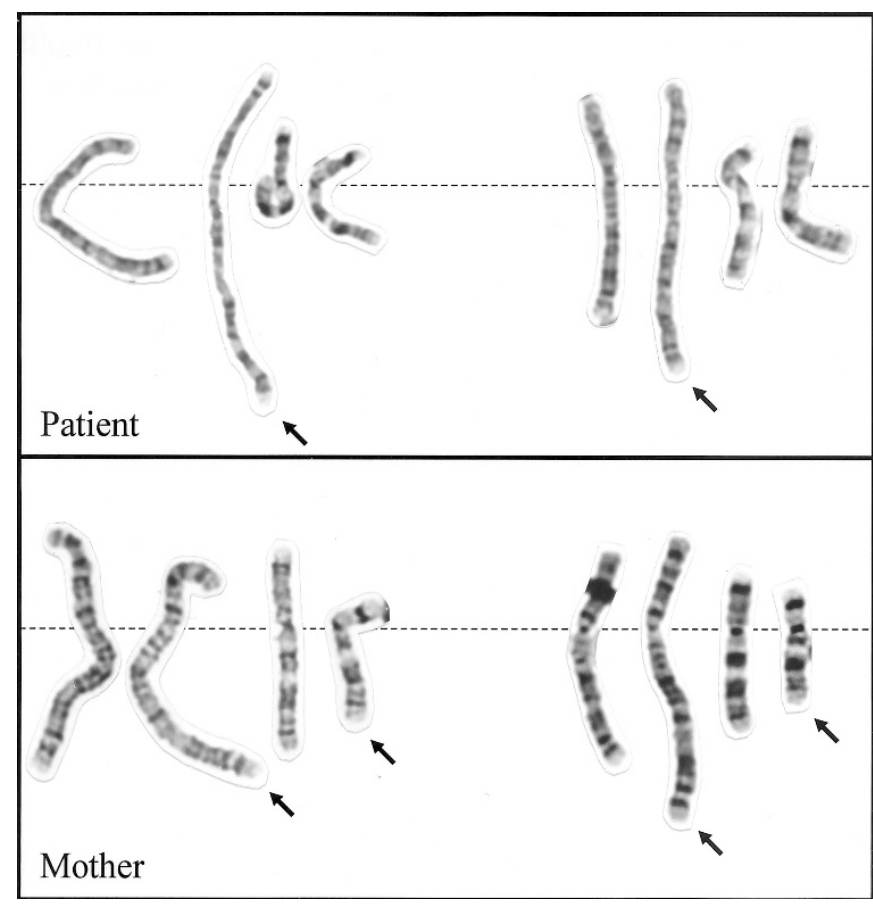

Fig. 2 Partial karyotypes showing the chromosome 2 and 7 homologues from two cells of the patient and her mother. Arrows indicate the abnormal chromosomes.

\section{RESULTS}

\section{Cytogenetic analysis}

High resolution chromosome analysis of the patient revealed an abnormal chromosome 2 with material of uncertain origin inserted into the long arm (Fig. 2). The patient's mother was found to have the same abnormal chromosome 2 as her daughter but also had a rearranged chromosome 7 (Fig. 2). Our original interpretation of the balanced rearrangement in the mother, based on GTG-banding alone, was that it was derived from an initial pericentric inversion in chromosome 7 with breakpoints at bands 7p15.3 and 7q11.23 followed by the insertion of material from both arms of the inverted chromosome 7 (7q21.2 $\rightarrow 7 q 21.1:: 7$ p15.3 $\rightarrow 7$ p13) into the long arm of chromosome 2 at band $2 \mathrm{q} 31$. Analysis with BAC clones did not support this interpretation and showed that the inserted material came only from the short arm of chromosome 7 (see below). The father and the maternal grandparents had normal karyotypes.

\section{Molecular cytogenetic analysis}

The painting probes, WCP2 and WCP7, specific for chromosomes 2 and 7, respectively, were hybridized to metaphase chromosome preparations from the patient's mother. WCP2
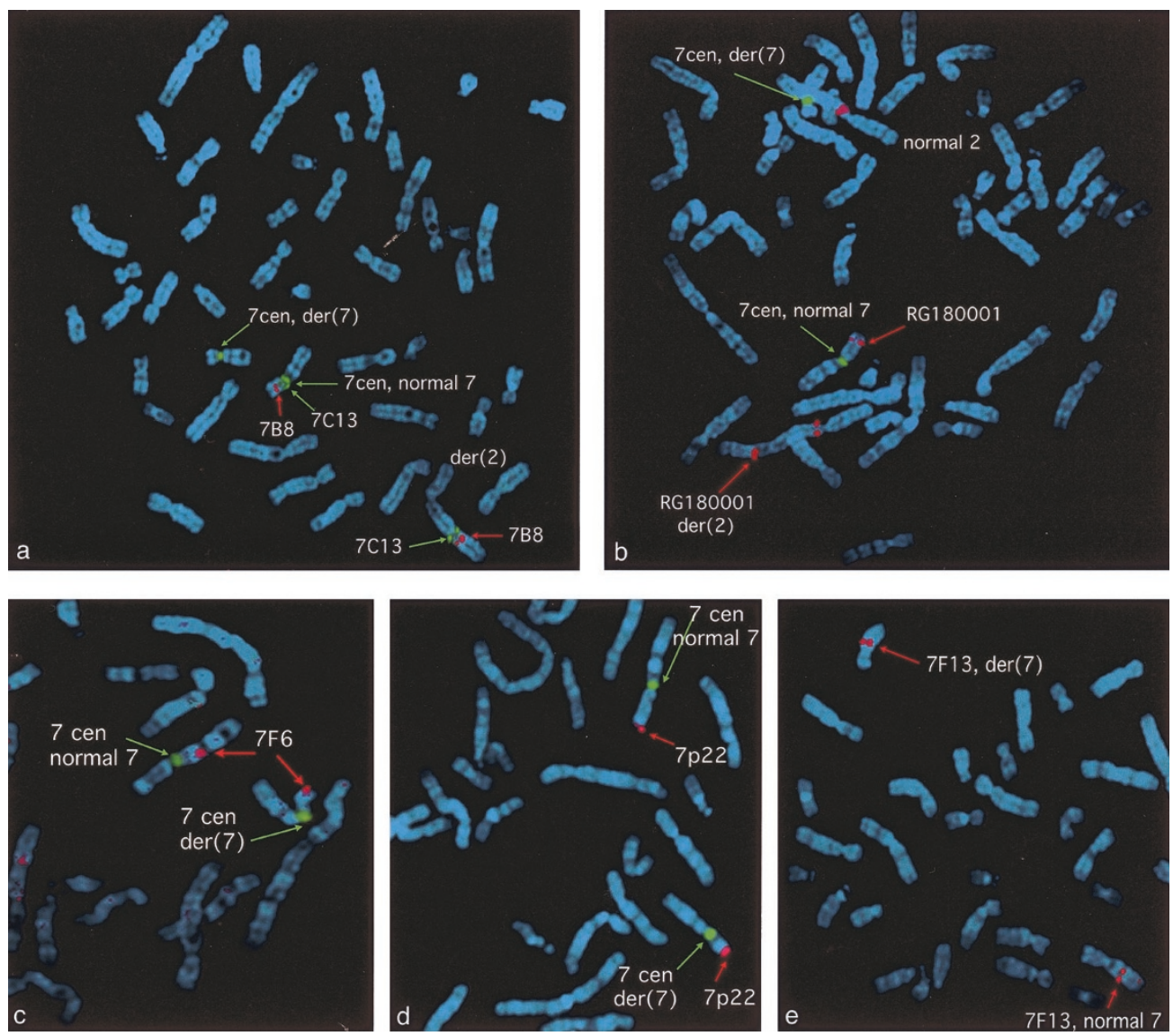

Fig. 3 FISH analysis of the mother's chromosomes. (a) BAC clones 7C13 (green) (mapping to 7p13), $7 \mathrm{~B} 8$ (red) (7p15.3 $\rightarrow 21.1)$, and the reference probe CEP 7 (green)(7cen); (b) BAC clone RG180O01 (red) (7p21.1 $\rightarrow$ p21.2) (note: this probe cross-hybridizes to the proximal long arm of chromosome 2); (c) BAC clone 7F6 (red) (7q21.2) with reference probe CEP 7 (green) (7cen); (d) distal 7p probe, D7S589 (red) (7p22) with reference probe CEP 7 (green) (7cen); (e) BAC clone 7F13 (red) (7q22). 


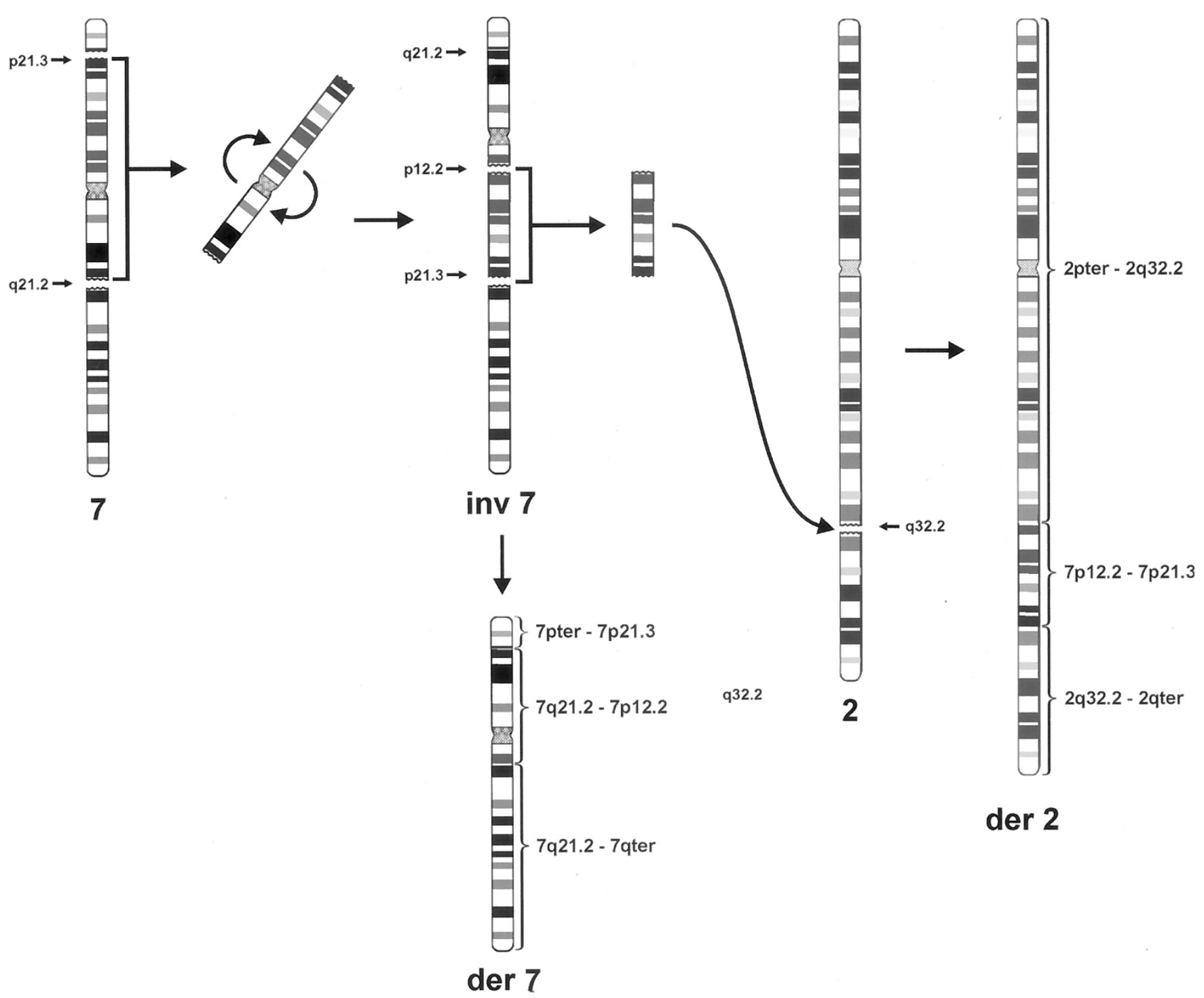

Fig. 4 Proposed mechanism of origin of the CCR in the patient's mother.

hybridized only with the normal and derivative chromosomes 2 , showing that no material from chromosome 2 was translocated to the der 7. WCP7 hybridized to the normal and der 7, as well as to the der 2 proving the chromosomal origin of the insertion (data not shown).

BAC clones mapping to the short arm of chromosome 7 from $7 \mathrm{p} 13 \rightarrow \mathrm{p} 21.2$ hybridized to the der 2, maintaining their original orientations to the centromere (direct insertion), as shown in Figure 3a and b. No probes mapping to the long arm of chromosome 7 hybridized to the der 2. Probes mapping to the proximal long arm of chromosome 7 from $7 \mathrm{q} 11.23 \rightarrow \mathrm{q} 21.1$ hybridized to the short arm of the der 7, confirming the pericentric inversion (Fig. 3c, and data not shown). A probe for 7 p22 (D7S589) hybridized to the distal short arm of the der 7 (Fig. 3d), while BAC RG180O01 which maps to 7p21.1 $\rightarrow$ p21.2 was inserted into the long arm of the der 2 (Fig. 3b), confirming the location of the inversion breakpoint, as well as one of the insertions breakpoints, in the short arm as proximal to
$7 \mathrm{p} 22$ and distal to $7 \mathrm{p} 21.1 \rightarrow \mathrm{p} 21.2$. BAC 7F13, which maps to $7 \mathrm{q} 22$, hybridized to the long arm of the der 7 (Fig. 3e), while BAC 7F6, mapping to 7q21.2, was inverted into the short arm of the der 7 (Fig. 3c), confirming the location of the inversion breakpoint in the long arm as distal to $7 \mathrm{q} 21.2$ and proximal to 7q22. BACs 7C13, which maps to 7p13, 7B8, which maps to $7 \mathrm{p} 15.3 \rightarrow \mathrm{p} 21.1$, and RG180O01, which maps to $7 \mathrm{p} 21.1 \rightarrow \mathrm{p} 21.2$, were all inserted into the long arm of the der 2 showing that the insertion extends at least from $7 \mathrm{p} 13$ to 7p21.1-p21.2. These data, in combination with high resolution chromosome analysis, indicate that the insertion probably extends from $7 \mathrm{p} 12.2$ to $7 \mathrm{p} 21.3$ and that that portion of the short arm of chromosome 7 is trisomic in our patient.

Because the patient had vertical plantar furrows similar to those seen in patients with trisomy 8 mosaicism, we performed FISH analysis using a chromosome painting probe specific for chromosome 8 . The probe painted only the chromosome 8 homologues and did not hybridize to the patient's abnormal 


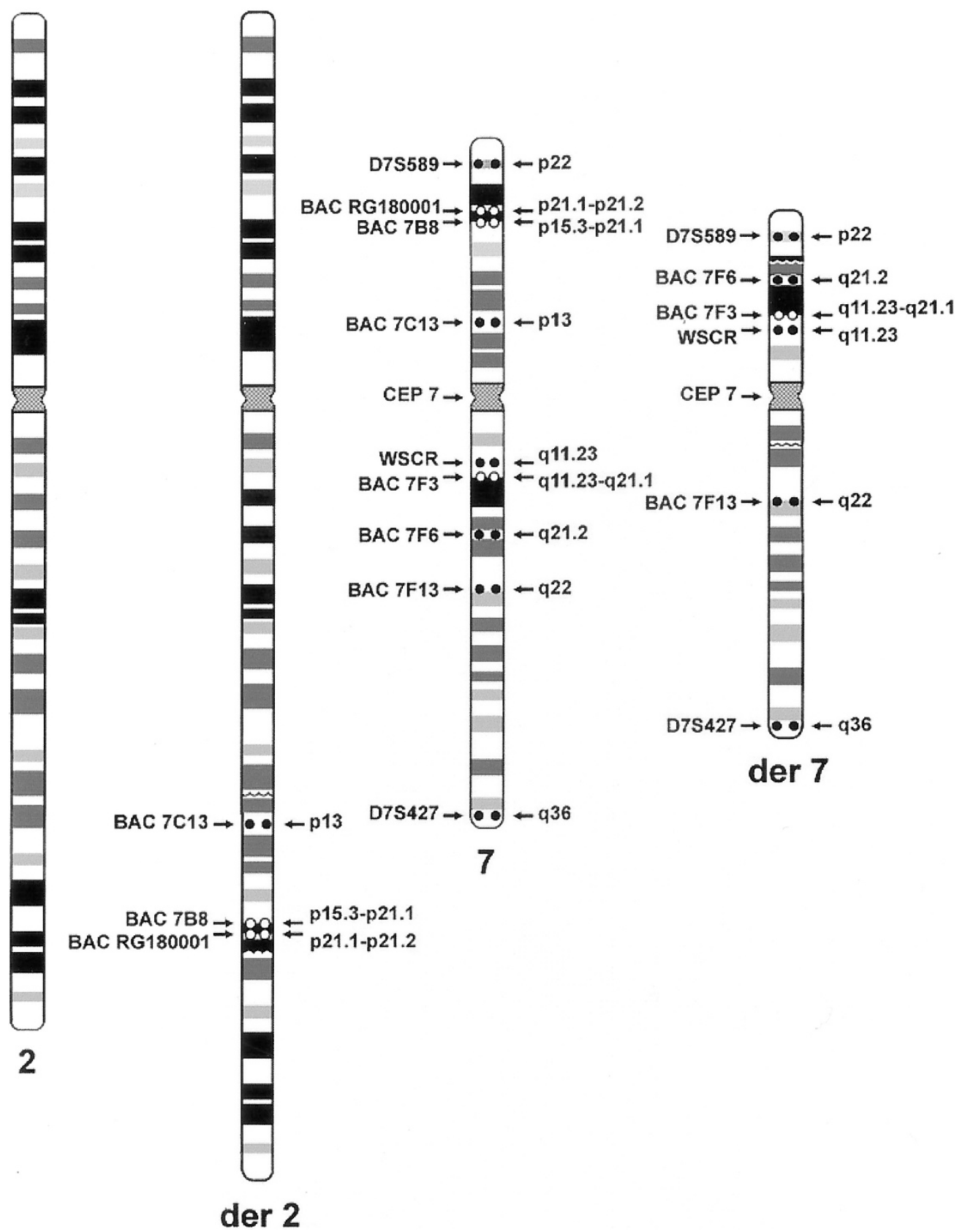

Fig. 5 Idiogram showing results of molecular cytogenetic studies of the mother's CCR. Filled or unfilled circles (for better visualization against negative or positive G-bands) indicate probe locations. Probe names are shown to the left of each chromosome; their corresponding map positions are indicated to the right of each chromosome.

chromosome 2 (data not shown), providing no evidence of partial trisomy 8 .

\section{DISCUSSION}

Cytogenetic and FISH analysis showed that the maternal CCR resulted from an initial pericentric inversion in chromosome 7 followed by insertion of a segment from the inverted chromosome 7 into the long arm of chromosome 2 (Fig. 4). Our initial interpretation of the rearrangement based on high resolution GTG-band analysis proposed an insertion of mate- rial from both arms of the inverted chromosome 7, including $7 \mathrm{p} 13 \rightarrow 7 \mathrm{p} 15.3$ and $7 \mathrm{q} 21.1 \rightarrow 7 \mathrm{q} 21.2$, into chromosome 2 . Although FISH results using the commercially available probes D7S589, WSCR, and D7S427 confirmed the presence of the pericentric inversion, none of these probes hybridized to the insertion. Thus, we were unable to fully characterize the rearrangement without the use of the panel of band-specific BAC probes. Our initial interpretation of the rearrangement was not supported by the results of FISH analysis using the BAC clone panel. No material from the long arm of chromosome 7 , including the predicted region, was detected within the insertion. 
Three BACs mapping to the short arm of chromosome 7 did hybridize to the der 2 (Fig. 5). We conclude that the inserted material is derived entirely from the short arm of chromosome 7 and that the CCR may involve only four breakpoints, although it cannot be assumed that the $7 \mathrm{p} 21.3$ inversion break point is the same as the $7 \mathrm{p} 21.3$ insertion breakpoint at the molecular level. Our final interpretation of the CCR in the mother is as follows: $\operatorname{ins}(2 ; 7) \operatorname{inv}(7)(2 \mathrm{pter} \rightarrow 2 \mathrm{q} 32.2:: 7 \mathrm{p} 12.2 \rightarrow 7 \mathrm{p} 21.3:: 2 \mathrm{q} 32.2 \rightarrow$ 2qter;7pter $\rightarrow 7 \mathrm{p} 21.3:: 7 \mathrm{q} 21.2 \rightarrow 7 \mathrm{p} 12.2:: 7 \mathrm{q} 21.2 \rightarrow 7 \mathrm{qter})$.

Through the use of the BAC clone panel, the chromosome abnormality in our patient was conclusively identified as a partial trisomy of the short arm of chromosome 7 (7p12.2 $\rightarrow 7 \mathrm{p} 21.3)$. Full or partial trisomy (duplication) $7 \mathrm{p}$ has been reported previously in 47 cases (for recent reviews see Cai et al., 1999; Pallotta et al., 1996; Reish et al., 1996; Lurie et al., 1995; Schaefer et al., 1995).4-8 Although the extent of the duplication varies among patients, a characteristic phenotype has emerged..$^{9}$ Among the common features are craniofacial anomalies, including dolichocephaly or brachycephaly; large fontanels; large, low-set, malformed ears; hypertelorism; down-slanting palpebral fissures; high or prominent forehead; broad or prominent nasal bridge; micrognathia; high arched palate; hyperextensible joints; cardiac defects; and psychomotor retardation. Reish and colleagues ${ }^{6}$ restricted the critical region for this phenotype to distal $7 \mathrm{p}(7 \mathrm{p} 15 \rightarrow \mathrm{pter})$ based on comparisons among 21 patients. They concluded that duplication $7 \mathrm{p} 15 \rightarrow$ pter produces the same phenotype as duplication of the entire short arm.

Our patient has most of the features ascribed to the trisomy $7 \mathrm{p}$ "critical region," although, her partial trisomy does not include to the distal half of the critical region ( $7 \mathrm{p} 21.3 \rightarrow 7 \mathrm{pter})$. There have been only six other reports of interstitial duplications of $7 \mathrm{p}^{8,10-14}$ In two of these patients, the duplication does not overlap the critical region. Schaefer et al. ${ }^{8}$ reported a child with $\operatorname{dup}(7)(\mathrm{p} 13 \rightarrow \mathrm{p} 12.2)$ who had failure to thrive, developmental delay, a mildly beaked nose, and clinodactyly. Her mother, grandmother, and one brother also had the duplication and were mildly retarded. No other features associated with trisomy $7 \mathrm{p}$ were reported. Šubrt et al. ${ }^{14}$ and S̆ubrt and Hníková ${ }^{15}$ also reported a patient whose duplication $(7 \mathrm{p} 13 \rightarrow \mathrm{p} 14)$ does not overlap the critical region. The patient had a "bird-like" face, microcephaly, hypertelorism, "gothic" palate, protruding ears, mental retardation, and cardiac anomalies.

The other patients with interstitial duplications of $7 \mathrm{p}$, including our patient, all have duplications overlapping the critical region and all show many of the clinical abnormalities ascribed to the critical region. Although none of the patients with interstitial duplications were trisomic for segment 7 p22.2 $\rightarrow 7$ pter of the critical region, patient variability prevents further narrowing of the critical region. However, the patient described by Cantú et al. ${ }^{13}$ and our patient have all of the clinical abnormalities of full trisomy $7 \mathrm{p}$ with the exception of dolichocephaly or brachycephaly and wide fontanels in our patient (initially examined at age 11), and cardiac anomalies, which were absent in both patients. On the other hand, cardiac anomalies were present in two other patients with interstitial duplications, one ${ }^{10}$ whose duplication $(7 \mathrm{p} 21.2 \rightarrow \mathrm{p} 22.1)$ ex- tends more distally than any other patient with an intersitial duplication, and the patient described above ${ }^{14,15}$ whose duplication is more proximal $(7 \mathrm{p} 13 \rightarrow \mathrm{p} 14)$.

Vertical plantar furrows, often observed in patients with trisomy 8 mosaicism, were also noted in our patient. To our knowledge this finding has not been reported previously in patients with trisomy $7 \mathrm{p}$.

The importance of FISH technology in characterization of CCRs is widely recognized. FISH permits visualization of chromosomal rearrangements at a sensitivity beyond that which can be achieved with high resolution banding. Through the use of a panel of BAC clones as probes, we were able to accurately characterize the balanced CCR found in our patient's mother and, therefore, were able to identify conclusively the region of chromosome 7 that is trisomic in our patient. Use of BAC clone panels, as illustrated here, should further enhance the diagnostic power of FISH technology.

\section{Acknowledgments}

This work was supported in part by Department of Energy grant 96ER6229A (J.R.K.) and National Institutes of Health grant HD33113 (J.R.K.). J.R.K. holds the Geri and Richard Brawerman Chair in Molecular Genetics.

\section{References}

1. Batista DAS, Tuck-Muller CM, Martinez JE, Kearns WG, Pearson PL, Stetten G. A complex chromosomal rearrangement detected prenatally and studied by fluorescence in situ hybridization. Hum Genet 1993;92:117-121.

2. Yunis JJ. High resolution of human chromosomes. Science 1976;191:1268-1270.

3. Korenberg JR, Chen XN, Sun Z, Shi ZY, Ma S, Vataru E, Yimlamai D, Weissenbach JS, Shizuya H, Simon MI, Gerety SS, Nguyen H, Zemsteva IS, Hui L, Silva J, Wu X, Birren BW, Hudson TJ. Human genome anatomy: BACs integrating the genetic and cytogenetic maps for bridging genome and biomedicine. Genome Res 1999;9:9941001 .

4. Cai T, Yu P, Tagle DA, Xia J. Duplication of 7p21.2 $\rightarrow$ pter due to maternal $7 \mathrm{p} ; 21 \mathrm{q}$ translocation: implications for critical segment assignment in the $7 \mathrm{p}$ duplication syndrome. Am J Med Genet 1999;86:305-311.

5. Pallotta R, Dalprà L, Fusilli $\mathrm{P}$, Zuffardi O. Further delineation of $7 \mathrm{p}$ trisomy. Case report and review of literature. Ann Genet 1996;39:152-158.

6. Reish O, Berry SA, Dewald G, King RA. Duplication of 7p; Further delineation of the phenotype and restriction of the critical region to the distal part of the short arm. Am J Med Genet 1996;61:21-25.

7. Lurie IW, Schwartz MF, Schwartz S, Cohen MM. Trisomy 7p resulting from isochromosome formation and whole-arm translocation. Am J Med Genet 1995;55:6266.

8. Schaefer GB, Novak K, Steele D, Buehler B, Smith S, Zaleski D, Pickering D, Nelson M, Sanger W. Familial inverted duplication 7p. Am J Med Genet 1995;56:184-187.

9. Milunsky JM, Wyandt HE, Milunsky A. Emerging phenotype of duplication (7p): a report of three cases and review of the literature. Am J Med Genet 1989;33:364-368.

10. Franz HBG, Schliephacke M, Niemann G, Mielke G, Backsch C. De novo direct tandem duplication of a small segment of the short arm of chromosome 7 (p21.22 $\rightarrow 22.1$ ). Clin Genet 1996;50:426-429.

11. Kleczkowska A, Decock P, van den Berghe H, Fryns JP. Borderline intelligence and discrete craniofacial dysmorphism in an adolescent female with partial trisomy $7 \mathrm{p}$ due to a de novo tandem duplication 7 (p15.1 $\rightarrow$ p21.3). Genet Couns 1994;5:393-397.

12. Dẹbiec-Rychter M, Overhauser J, Kałuźewski B, Jakubowski L, Truszczak B, Wilson W, Skorski M, Jackson L. De novo direct tandem duplication of the short arm of chromosome 7(p21.1 $\rightarrow$ p14.2). Am J Med Genet 1990;36:316-320.

13. Cantú JM, Rivas F, Ruiz C, Barajas LO, Moller M, Rivera H. Trisomy 7p due to a mosaic normal/dir dup (7) (p13 $\rightarrow$ p22). Syndrome delineation, critical segment assignment, and a comment on duplications. Ann Genet 1985;28:254-257.

14. Šubrt I, Kozák J, Hníková O. Microdensitometric identification of the pericentric inversion of chromosome no. 2 and of duplication of the short arm of chromosome no. 7 in a reexamined case. Hum Hered 1973;23:331-337.

15. Subrt I, Hníková O. Chromosomal abnormalities in a girl with physical and mental maldevelopment. Hum Hered 1970;20:252-259. 